\title{
SOBRE LA AMBIGÜEDAD SEMÁNTICA EN LA INTERPRETACIÓN JURÍDICA
}

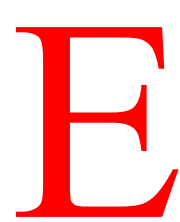

1 uso de la ambigüedad semántica intencional en la literatura puede remontarse a los días de la Antigua Grecia. Los términos asíndeton, parataxis, y elipsis son una prueba de ello. Asíndeton significa la omisión de conjunciones. En el caso de la parataxis, no se usa ninguna conjunción conectiva entre enunciados. La omisión de nombres, pronombres, verbos que no están en infinitivo, u otras partes sintácticas del lenguaje se incluyen en la elipsis. La utilización de estos medios estilísticos, sin embargo, sólo está permitido si el significado del texto no se desvirtúa con ello. ${ }^{1}$

Un fenómeno similar se encuentra también en textos jurídicos. En el contexto jurídico, sin embargo, la imprecisión semántica no se debe a un fin estético. Ni siquiera se pretende en muchos casos. Los textos jurídicos tienen lagunas; es decir, no dan, o mejor no pueden dar respuesta a todas las cuestiones que sólo se materializarán posteriormente. A pesar de las obvias diferencias, una comparación teórica de la ambigüedad semántica en textos jurídicos, por una parte, y en la literatura por la otra, parece ser un desafío tentador. Acepto el desafío ayudado por el tratado de Ms. Leena Kirstinä, «Epämääräisyyskohta teoksen rakenteen ominaisuutena Roman Ingardenin ja Wolfgang Iserin mukaan» ${ }^{2}$ («La ambigüedad semántica como una característica de una obra de arte literaria según Roman Ingarden y Wolfgang Iser»).

Ms. Leena Kirstinä ha analizado profundamente el poema «Vävy» («Yerno») de Einari Vuorela. Este pequeño poema de ficción describe una historia completa en seis versos: un vagabundo en su caminar a lo largo de bosques llega a una pequeña casa, en la que encuentra, con gran contento, a una joven y su madre. La atmósfera resulta cómoda y al despuntar el nuevo día, el caminante se da cuenta de que se ha convertido en yerno. El autor, Mr. Vuorela, ha usado imprecisiones semánticas como un medio estilístico efectivo. La historia se desarrolla entre líneas, y el texto escrito contiene ambigüedad semántica, que el lector tiene que resolver por sí mismo.

${ }^{1}$ Kirstinä, Leena, Epämääräisyyskohta teoksen rakenteen ominaisuutena Roman Ingardenin ja Wolfgang Iserin mukaan («Sobre la ambigüedad semántica como una característica de una obra de arte literaria según Roman Ingarden y Wolfgang Iser»). Tampereen Yliopisto. Yleinen kirjallisuustiede. Monistesarja N. ${ }^{\circ} 17,1984$, p. 3-4 (citado en adelante como Kirstinä).

${ }^{2}$ En cuanto a los fundamentos filosóficos véase también Kirstina, Leena, Aika, tarkastelukeskus ja kerronnan rakenne Claude Simonin romazinituotannossa («Tiempo, Lugar de Observación y estructura de la narrativa en la producción novelística de Claude Simon»). Tampereen Yliopisto. Kirjallisuustieteen julkaisuja n. ${ }^{\circ} 15,1981$. 
Ms. Leena Kirstinä hace referencia a la teoría presentada por Roman Ingarden, que se centra en el concepto de ambigüedad semántica y su significación en la interpretación. ${ }^{3}$ Wolfgang Iser ataca la misma cuestión analizando la comunicación entre el lector y el texto. El objetivo de Iser es analizar la estructura del texto y sus efectos sobre el lector. El concepto clave de Iser es la ambigüedad semántica, que hace referencia a una laguna entre las partes características del texto. Tal laguna obliga al lector a completar el texto de forma coherente, y así, en cierta manera a reconstruir los contenidos imaginados de la obra en cuestión. No obstante, la ambigüedad semántica de Ingarden no está relacionada con el texto mismo, sino que es parte de los objetos, personas, sucesos y situaciones del mundo que la obra literaria describe. En este aspecto, una comparación con el concepto de laguna jurídica es digno de anotarse.

La noción de ambigüedad semántica de Iser es paralela al concepto de laguna jurídica: el texto guarda silencio sobre la cuestión planteada y el intérprete (lector) tiene que llenar la laguna con otros materiales. La ambigüedad semántica, que es esencial para la teoría de Ingarden, está estrechamente relacionada con la laguna en un sistema jurídico. ${ }^{4} \mathrm{La}$ laguna no está en el texto, sino, por así decir, en los asuntos de los que trata el texto.

Roman Ingarden desarrolla además la teoría estableciendo que una obra literaria es siempre un tipo de esquema -no en el sentido de estar inacabado, sino en el de estar abierto a varias concreciones diferentes. ${ }^{5}$ El lector construye estas concreciones al ir leyendo, al especificar e implementar las ambigüedades semánticas de acuerdo con las sugerencias (factores orientativos) que están dados en el texto. Al lector, en cierta forma, se le pone en la tesitura de tener que dar vida a ciertos aspectos del texto. Las ambigüedades semánticas no se reconocen, sin embargo, al ir leyendo. Sólo son reveladas en un posterior análisis. Por consiguiente, Ingarden habla de la reconstrucción de la obra literaria.

Leena Kirstinä ilustra este proceso de reconstrucción con la ayuda del concepto de rompecabezas. Escribe. «Una obra de arte literaria se diferencia de un rompecabezas por lo menos en un aspecto importante. Puede ser completada de varias formas diferentes, es decir, su significado puede concretarse de varias maneras. Por consiguiente, no se puede presuponer que debe existir sólo un 'significado' (fijo) de la obra». ${ }^{6}$

${ }^{3}$ Véase Kirstinä, p. 5-6. Todas mis referencias a Ingarden e Iser son indirectas, es decir, basadas en el tratado de Leena Kirstinä. Para la finalidad de este ensayo, sin embargo, la extensión de la fuente es suficiente.

${ }^{4}$ Sobre los conceptos de laguna jurídica y laguna en el sistema jurídico, véase por ejemplo, Brusiin, Otto, Tuomarin harkinta normin puuttuessa («Discreción jurídica en ausencia de una norma»), 1938, p. 24 ss.; y Makkonen, Kaarle, Zur Problematik der juridischen Entscheidung», 1965, p. 122 ss.

${ }^{5}$ Kirstinä, p. 12 ss.

${ }^{6}$ Kirstinä, p. 53. 
Lo que es importante es que los rasgos artísticos y estéticos se valoran contrastados con la estructura de un significado concreto en un tiempo. Este significado particular es un tipo de fundamento que unifica los elementos de ese esquema de interpretación particular.

La teoría de Ingarden tiene una dificultad inherente, que Leena Kirstinä establece como sigue: los diversos significados concretados parecen dar la impresión de que la misma obra se disolviera en experiencias de carácter subjetivo. ${ }^{7}$ La dificultad es obvia, si aceptamos la noción de que una obra de arte está abierta a varias y diferentes reconstrucciones, cuyos contenidos reposan en último término sobre los lectores individuales ¿Es posible seguir hablando de la reconstrucción de la misma obra? Con referencia a las ideas de Ingarden, Leena Kirstinä sin embargo apunta que en cada significado concretado del texto se preserva la misma estructura básica. Algunas de las concreciones están más cerca del texto original, otras más lejos. Aun así, está bien justificado el preguntar cómo se distinguen las reconstrucciones erróneas de las correctas. Ingarden fundamenta su propia respuesta sobre la función de la literatura. Para él, la función principal de la literatura es estética, y así todas las obras literarias que no cumplan este criterio no entran en su concepción de la literatura. ${ }^{8}$ Parece permisible pensar que teniendo en cuenta esta noción, las falsas reconstrucciones son parcialmente distinguidas de las correctas. Esto, sin embargo, no resuelve todo el problema. Según Kirstinä, Ingarden tiende a pensar que los así llamados falsos hábitos de lectura son parcialmente determinados por la situación cultural. El medio cultural y la comunidad de lectores influyen sobre qué clase de concreción sobrevivirá. Kirstinä además añade que Ingarden no desarrolla más la teoría de la relación entre la función estética de la literatura y la sociedad. ${ }^{9}$

En su lugar, Ingarden ofrece otros medios de reconocer lo estéticamente significativo, o las concreciones de significado «correctas». Opina que los lectores profesionales de la literatura deben analizar completamente la obra. Así, resultarán un gran número de significados concretos analizados por los profesionales. En último término, el auditorio erudito representa los criterios de corrección e incorrección en el esquema de Ingarden. ${ }^{10}$

La noción es muy similar a la teoría de la interpretación jurídica. Los filósofos del derecho han estado perplejos durante mucho tiempo por la cuestión de cuál es la única solución correcta en cada situación en la que se toma una decisión jurídica. Algunos han argumentado que tal solución siempre existe -aún en los así llamados casos difíciles-;

${ }^{7}$ Kirstinä, p. 58-59.

${ }^{8}$ Kirstinä, p. 59.

${ }^{9}$ Kirstinä, p. 60.

${ }^{10}$ Kirstinä, p. 62. 
y también que tal solución puede, al menos en principio, ser encontrada. Otros han negado la posibilidad de hallar tal solución, así como su existencia. ${ }^{11}$ La teoría europea de la interpretación jurídica ha defendido generalmente la última opinión. En Finlandia, el Dr. Kaarle Makkonen ha sido el defensor de esta concepción. En su tesis doctoral, argumentó poderosamente contra la posibilidad de la única solución correcta, presentando argumentos de tipo lingüístico, lógico y jurídico. ${ }^{12}$

Yo mismo he utilizado la ilustración del rompecabezas en la interpretación jurídica, de forma bastante similar a la de Kirstinä al analizar la estructura de una obra de arte literaria. Cuando el texto jurídico que se examina contiene ambigüedad semántica (en el sentido señalado por Iser o Ingarden), el intérprete tiene que buscar la información necesaria fuera del texto. Las partes de información necesaria son como las piezas de un rompecabezas. La diferencia significativa consiste en que la forma del «rompecabezas jurídico» no se conoce antes de completarse. Las semejanzas con la interpretación de una novela son aparentes. Entre tanto, la diferencia significativa entre estas dos debe señalarse.

Una obra de arte literaria puede ser reconstruida de varias formas dependiendo de cómo el lector es guiado por los factores orientativos dados por el autor. En principio, no existen fundamentos o normas de autoridad, que delimiten la línea divisoria entre las concreciones «permitidas» y las «prohibidas». Como se ha establecido antes, éste parece ser el punto débil en, por ejemplo, la teoría de Ingarden. Está obligado a introducir un tipo de escala valorativa, que es creada y se mantiene por el así llamado auditorio de lectores profesionales. La interpretación jurídica, por otra parte, se caracteriza por la existencia de fundamentos de autoridad para la interpretación (fuentes del derecho) así como de principios que rigen su utilización, que han sido interiorizados por la comunidad jurídica. ${ }^{13}$ Estos principios que rigen el proceso de interpretación tienen su origen o en los textos jurídicos o en la praxis jurídica. De hecho, está justificada una tesis más fuerte: una interpretación puede ser debidamente llamada jurídica si, y sólo si se basa en las fuentes del derecho autorizadas, y se han respetado los principios reconocidos del proceso de interpretación jurídica. A causa de estos dos factores es decir, las fuentes del derecho y los principios que rigen su utilización la ciencia jurídica y la interpretación jurídica se distinguen en general de otras ramas del conocimiento e investigación científica, por ejemplo, la sociología, historia o los estudios de literatura. Y los mismos principios diferencian

${ }^{11}$ Sobre estas doctrinas, véase Aarnio, Oikeussäännösten tulkinnasta, 1982, p. 150 ss.

${ }^{12}$ Makkonen, Zur Problematik der juridischen Entscheidung, p. 214 ss. Véase también Oker-Blom, Max, «En enda riktig lösning trots allt? Dworkin mot finländsk bakgrund», JFT 1978, p. 251 ss.

${ }^{13}$ Véase Aarnio, Oikeussäännösten tulkinnasta, p. 28 ss., donde se tratan las así llamadas pre-condiciones de un paradigma jurídico. 
la interpretación jurídica de, por ejemplo, la interpretación de principios morales. ${ }^{14}$

La interpretación de una novela y la interpretación jurídica se consideran muy parecidas a causa de que ambas implican una reacción del lector a un mensaje escrito dado. Desde este punto de vista, la interpretación es esencialmente «dependiente del lector» en ambos casos. La teoría de Ingarden, me parece, es bastante típica al afirmar que la interpretación de una novela necesita no salir «fuera del lector». De más interés son, con todo, las teorías que perfilan una cierta clase de lectores ideales. Como un ejemplo de ello, Leena Kirstinä menciona al «super-lector», término colectivo que engloba a lectores de diferente competencia, teniendo todos ellos algo en común: entienden los puntos claves de la obra de la misma forma. Otra clase de lector ideal es el «lector informado», que puede ser definido como un lector que posee la máxima competencia. El «lector ideal» específico también pertenece a la misma categoría. ${ }^{15}$

Estos tipos ideales sin duda recuerdan al juez ficticio de Ronal Dworkin, «Hércules J.». Es un super-juez, omnisciente y omnipotente, y en principio siempre capaz de tomar la decisión correcta en una disputa jurídica. Tiene acceso al máximo de información, y utiliza esta información de una forma ideal, libre de todos los factores irrelevantes o aleatorios. Para ser capaz de buscar la sola y única solución del caso del que se trata, Hércules J. tiene que ser capaz también de hacer valoraciones objetivamente válidas. En otras palabras, Dworkin está forzado a comprometerse con el objetivismo respecto a los valores con el fin de que la construcción de su super-juez sea concebible. ${ }^{16}$

No estoy en la posición de estimar aquí las consecuencias completas o los pre-requisitos de la noción de «lector ideal». Sin embargo, la mera

${ }^{14}$ De hecho, podríamos ilustrar la interpretación lingüística con la ayuda de una línea recta, colocando los varios tipos de interpretación lingüística en esa línea. Podemos diferenciar entre, por ejemplo, la interpretación lingüística en sentido general, interpretación de una novela, de un testamento o un contrato, de un texto jurídico, libre discrecionalidad en la administración y la interpretación de principios morales. Los distintos tipos de casos de interpretación pueden distinguirse con respecto al material de la fuente y los principios (o reglas) que rigen su uso en la interpretación que están permitidos en cada caso. Así, en un extremo de la línea colocamos la interpretación lingüística en sentido general, que normalmente no tiene restricciones en cuanto al material de las fuentes y su uso en la interpretación. En el centro de la línea colocamos la interpretación jurídica con sus diversas restricciones sobre el proceso interpretativo. Y en el otro extremo, existe la interpretación de los principios morales que goza de más libertad que su contrapartida jurídica. Al colocar la interpretación jurídica en el centro de la línea subrayamos sus características distintivas en esta familia de tipos de interpretación. Lo que merece notarse es que la separación entre los diversos tipos de interpretación no necesita ser muy nítida o clara; más bien el tránsito de un tipo a otro es muy suave.

${ }^{15}$ Kirstinä, p. 7 y ss. Y también p. 15 ss.

${ }^{16}$ Dworkin, Ronald, Taking Rights Seriously, 1977, por ejemplo. Véase también Aarnio, Oikeussäännösten tulkinnasta, p. 153 ss. y las referencias que allí se citan. 
comparación entre el lector ideal y Hércules J. es en sí misma reconfortante. Muestra cómo muy distintas ramas de investigación pueden hacer uso del mismo tipo de construcciones teóricas, que claramente denotan las semejanzas básicas en su punto de partida. Basado sobre argumentos que no puedo utilizar en este contexto, el Hércules J. de Dworkin puede mostrarse que es una idealización muy fuerte. Simplemente no puede cumplir la tarea para la que fue hecho. Una de las razones que contribuyen a ello, merece comentarse aquí. La teoría de Dworkin no presta bastante atención al papel del auditorio al que se dirige la interpretación. Las semejanzas con la problemática de Ingarden respecto a la relación entre un lector y la colectividad de lectores son de nuevo obvias. Por consiguiente, trataré con mayor consideración esta cuestión particular.

En la interpretación jurídica no sólo es significativa la relación entre el que emite el texto (es decir, el legislador) y el que lo interpreta (es decir, el juez o el funcionario). También tiene que tenerse en cuenta la relación que el intérprete tiene con los otros miembros del auditorio de la interpretación o comunidad jurídica. ${ }^{17} \mathrm{El}$ intérprete jurídico no puede aspirar a un resultado que sólo le satisfaga a sí mismo. En su lugar, tiene que llegar a un nivel de aceptabilidad general al atribuir un significado a una parte de un texto jurídico. Un proceso de comunicación siempre conlleva una interacción social: del legislador al intérprete $\mathrm{y}$, después, al auditorio de la interpretación; y viceversa. El flujo en las dos direcciones del proceso de la comunicación se basa en el hecho de que el Derecho es un medio autorizado de poder social, y los resultados de la interpretación determinan así el ejercicio del poder social en una sociedad dada. ${ }^{18}$ En consecuencia, el control de los resultados de la interpretación es bastante esencial en el campo del Derecho -y no en el campo del estudio de literatura-. Derecho, poder e interpretación constituyen una unión, que no tiene contrapartida en la interpretación de una novela.

Así, es fácil entender el papel crucial del auditorio jurídico (o comunidad) en la teoría de la interpretación jurídica. ${ }^{19}$ La interpretación jurídica es esencialmente un fenómeno social. Esta línea de argumentación nos retorna a la doctrina de la única solución correcta. Como en el caso de la interpretación de una novela, un teórico jurídico puede plantear

${ }^{17}$ Véase Aarnio, «Lainoppi -tiedettä vai tekniikkaa» («La doctrina jurídica -Ciencia o Técnica-»), Academia Scientiarum Fennica, Vuoisikirja 1978, p. 143 ss. Véase también Aarnio, Philosophical Perspectives in Jurisprudence. Acta Philosophica Fennica, Vol. 36, 1983, p. 47 ss. -Sobre la relación entre la interpretación jurídica y otros tipos de interpretación en general-, véase Tolkning och tolkningsteorier. Kungl Vilterhets Historie och Antikvitets Akademien. Konferenser 7, 1982, e.g. p. 3-84 y p. 141 ss.

${ }^{18}$ Véase la opinión de Kauko Wikström sobre el derecho y el poder en Wikström, Oikeuskäytännön tulkinnasta. Erittely oikeusnormien soveltamistoiminnasta esitettävien väitteiden teoreettisista perusteista ja oikeuskäytännön rakenteesta («Sobre la interpretación de la práctica jurídica»), 1979, p. 189 ss.

${ }^{19}$ Aarnio, Oikeuskäytärnnön tulkinnasta, p. 190 ss. 
también la siguiente cuestión: si la posibilidad de que se opongan diferentes interpretaciones alternativas socava las bases del Derecho. ¿Qué queda del concepto del Derecho, si una única norma jurídica puede adquirir contenidos diferentes a través del proceso de interpretación; $\mathrm{y}$, además, ninguna de las alternativas puede probarse que sea peor que las otras? La problemática en cuestión es la misma que Leena Kirstinä comenta como sigue: ¿es posible hablar sobre la misma obra de arte, si las diversas concreciones de la obra, por así decirlo, se dividen en diferentes experiencias subjetivas?

Para responder esta clase de preguntas puede parecer necesario introducir algún tipo de escala o medida con ayuda de la cual se puedan comparar entre sí las diferentes interpretaciones. Sin embargo, la comparación de las interpretaciones alternativas no pueden conducir al reconocimiento de la única solución apropiada al caso jurídico particular, puesto que esta doctrina ya se ha mostrado insatisfactoria. Así, nos enfrentamos con una necesidad de comparar entre las interpretaciones alternativas y con una bien justificada duda de que la comparación no conduce a la única solución correcta.

Se ha sugerido también un compromiso; el resultado de una interpretación tal que pueda conseguir la aceptación de la mayoría de un auditorio jurídico, es la alternativa más justificada (o «mejor»). Además, tiene que cumplirse una condición adicional: el auditorio jurídico en cuestión tiene que ajustarse a los principios del discurso racional al decidir el caso. El resultado de la interpretación no puede ser verdadero en el sentido de la teoría de la verdad como correspondencia, puesto que no existe ninguna realidad con la que el enunciado de la interpretación pueda ser contrastado. En su lugar, estamos tratando con una reconstrucción interpretativa, que crea su propia «realidad».

La pretensión de racionalidad en la interpretación jurídica no implica, sin embargo, que cada uno y cualquiera que se ajuste a los principios de la racionalidad deba racionalmente aceptar los mismos resultados de la interpretación. La interpretación jurídica siempre implica una selección entre alternativas. El número de interpretaciones alternativas finales puede ser reducida parcialmente con la ayuda de criterios lingüísticos (semánticos) -exactamente de la misma forma como cualquiera puede juzgar alguna interpretación «imposible»sobre la base de la propia competencia lingüística-. Además la reducción de interpretaciones alternativas puede basarse parcialmente sobre criterios jurídicos. Cada profesional de la rama jurídica puede determinar que cierta alternativa no está definitivamente de acuerdo con el Derecho.

En los casos difíciles, aún queda un espectro de interpretaciones alternativas, que no pueden reducirse más por medio de criterios lingüísticos o jurídicos convencionales. Pero, por ejemplo un juez se enfrenta con la obligación de tomar una decisión en cada una de las disputas jurídicas que se presentan ante el tribunal. Al tomar su «elección final», no puede ignorar las valoraciones relacionadas con el resultado de esa interpretación particular. Lo que está implícito es un orden preferencial de las valoraciones implicadas. Si aceptamos la noción de que a los valores 
les falta un carácter objetivo y que dos individuos pueden muy bien ser justificados en su desacuerdo sobre ciertos valores; entonces tenemos que reconocer la posibilidad de que un auditorio racional de la interpretación puede estar dividido al considerar la elección entre los distintos resultados de la interpretación. En tal situación, podrían muy bien no ser asequibles más criterios para clasificar las alternativas. Un criterio, con todo, es el principio democrático de la voluntad de la mayoría -con todas sus debilidades inherentes-. La interpretación alternativa, que la mayoría de la comunidad humana puede aceptar después de un discurso racional, no es ciertamente la única solución correcta, pero es lo más que se puede conseguir en esa situación.

El auditorio de la interpretación consistente únicamente de personas tales que se ajusten al principio del discurso racional es por supuesto un estado ideal de cosas. No existe en realidad ninguna de tales comunidades jurídicas. Sin embargo, la función de tal auditorio es sólo puramente teórica. Sirve como una medida o criterio para sopesar las diversas interpretaciones alternativas. Tal medida es de extrema importancia, especialmente en el campo del derecho. La jurisdicción no puede basarse sobre reglas arbitrarias. Cada ciudadano espera -y tiene el derecho de esperar- que su demanda de protección jurídica se respete cuando tiene que buscar justicia por medio de la maquinaria estatal. Si la jurisdicción se moviera en un ejercicio caprichoso y arbitrario de poder coercitivo, difícilmente podríamos hablar más de sistema jurídico en el sentido europeo.

Para diferenciar el derecho de la mera coerción social arbitraria necesitamos una medida o escala. Cada uno de tales criterios no es más que un ideal, como el modelo de un metro situado en París. Es tarea de la teoría jurídica proporcionar a la comunidad jurídica tales criterios de un sistema jurídico ideal. Con tal modelo ideal, podemos intentar su realización en nuestra vida social. El elemento esencialmente ideal de esta noción es muy semejante al concepto de verdad en las ciencias naturales. También, es un ideal que dirige el curso de la investigación científica de cada día -sin necesariamente investigar este objetivo.

Para cumplir las necesidades de interacción social y el ejercicio jurídico del poder social, es suficiente pretender un resultado que pueda ser reconocido por la mayoría de tal comunidad jurídica después de considerar el asunto racionalmente. La comunidad jurídica (auditorio) no tiene aquí el mismo contenido que el auditorio de Ingarden de lectores profesionales. El auditorio jurídico está compuesto por todos los individuos que tienen interés en tomar parte en el discurso sobre las interpretaciones alternativas. El auditorio de la interpretación tampoco es igual que el lector ideal de cualquier tipo, si tal construcción conlleva la doctrina de la única solución correcta. La comunidad jurídica en cuestión es esencialmente «relativista», en el sentido de admitir la posibilidad de desacuerdo sobre valoraciones.

Cuando se reúnen todas las semejanzas y factores de diferenciación, lo que queda es la semejanza estructural entre la interpretación de una novela y la jurídica. La presencia de ambigüedad semántica, en el sentido 
atribuido a Ingarden e Iser, es típico de ambas; han de tolerarse diversas reconstrucciones alternativas. Muy generalmente, el proceso de interpretación está dirigido por factores de un tipo similar. Y creo que las ontologías de la interpretación jurídica y la interpretación de una novela tienen también algo en común.

La referencia a lo autorizado en la interpretación jurídica es la característica distintiva más significativa entre las dos. Los objetos de la interpretación jurídica son los textos promulgados oficialmente, cuya función es servir como instrumentos en el ejercicio del poder social. Por consiguiente, la interpretación jurídica está más atada que la interpretación de una novela en cuanto se refiere a las fuentes y los principios que rigen su uso. A pesar de ello, la teoría de la interpretación de una novela parece ofrecer varias ideas nuevas a la teoría jurídica. Pienso, en particular, en el análisis del proceso de interacción, que ha sido ampliamente estudiado por los eruditos de la literatura. Además, el cambio de ideas no necesita ser unilateral. Por ejemplo, en la teoría de la novela el concepto y estatus del auditorio de la interpretación se ha demostrado problemático en muchos aspectos. En este contexto, la teoría jurídica podría tener algo que ofrecer a la teoría de la literatura. En resumen, la imprecisión semántica intencional, usada como un medio estilístico en literatura, tiene mucho en común con su contrapartida jurídica, la ambigüedad semántica no pretendida en los textos jurídicos.

(Traducción de José Pedro Úbeda) 